\title{
Identifying the morphological growth patterns of microbiological data types using computer-vision and statistical modelling
}

\author{
Sabeeha Sultana \\ Asst Professor,Dept of Computer Science, Jain University,Bangalore \\ Mohammad Basha \\ Senior Scientist,R\&D,Kumar Organic Pvt Ltd, Jegani,Bangalore
}

An automatic tool is industrialized to recognize and classify morphological growth patterns of various microbiological data types by means of computer-vision and statistical modelling techniques. In algae phage (phage) typing, representative profiles of morphological growth stages of different algae types are extracted. Present systems rely on the subjective reading of the profiles of the by a human expert, which is time-consuming and prone to errors. The statistical methodology presented in this work, provides for an automated, objective and robust analysis of the visual data, along with the ability to cope with increasing data volumes. Validation is performed by comparison to an expert manual segmentation and labelling of the phage profiles. The statistical analysis such as the descriptive statistics is performed on time series data extracted is important for understanding relationships between parameters, provides insight into the growth curve of microalgae and cyanobacteria (correlation) and an essential step to estimate yield of biomass, etc., or predict the duration to achieve a certain yield of a pigment or protein, etc., for commercial applications. There are numerous methods for modelling time series data and being able to predict specific values and ; specifically, Regression Analysis and Analysis of Variance (ANOVA) are foremost among them. Computation of the correlation coefficient aids in better understanding the relationships that exist between various parameters that evolve with time and change with different phases of the growth of the organism (and cyanobacteria). This study focuses on statistical techniques for the analysis of time-series data.

Keywords: Cyanobacteria, Linear regression, Scatter plots, Correlation, Timeseries, Chlorophyll, GrowthPhases, Statistics, Errorbars, Biomass, Training model

\section{Introduction}

The cytology, which deals with the study of cells in terms of their origin, structure, organelles and functional properties, is of key importance in biology and medicine. It consists of the recognition of cell types, fundamental for understanding biological differentiation (e.g., discriminating organisms or cell types in the same tissue); In this study, we investigate the feasibility of leveraging machine learning and fluorescence-based spectral morphological features to enable the identification of six different algae types in an automated fashion. More specifically, we explore and investigate the efficacy of a number of different morphological and spectral fluorescence features extracted from multi-band fluorescence imaging data when used to train neural network classification models designed for the purpose of identification of algae types in an automated manner .Manual identification of species according to correct taxonomy is generally impeded by obstacles such as declining the number of taxonomists and the increase in the number of described species, also discussed in detail by Gaston and O'Neill [1] and Peterson [2], which makes identification of specimens to species a difficult 
and time consuming task. Automated tools may significantly assist in species recognition by facilitating reliable identification of any specimens in a population. Automated methods that rely on pattern recognition and image analysis have been widely applied for recognition and categorization of biological images in the field of biodiversity [3-12]. Content-based retrieval is one of the common text-based approaches in image retrieval domain [13-15] in a way that images of specimens are matched with images in a database according to visual content (color, shape, texture) similarities. For identification of species, visual features that are extracted from images based on morphology and taxonomic information play an essential role.

Many research works have been reported in the literature on this subject. The statistical image analysis for automatic identification of bacterial types are proposed in and Greenspan (2004)[16]. The artificial neural network approach for bacterial classification has been investigated (Nicholas et al., 1998)[17]. The data mining techniques are employed for the classification of HEp-2 cells in Petra (2001)[18] in which a simple set of shape features are used for classification of bacterial cells. Carolina et al. (2002) have investigated algorithms for cytoplasm segmentation of fluorescence-labeled cells using statistical analysis techniques based on shape descriptive features. A computer-aided system for the image analysis of bacterial in microbial communities using geometric shape features has been investigated in Liu et al. (2001) [19]. The automatic identification and classification of bacilli bacterial cell growth phases has been proposed in and (2010) using geometric shape features. A new image analysis tool to study biomass and of three major groups in an alpine lake using geometric features have been proposed in Thomas et al. (2009) [20]. An efficient automated method for image-based classification of microbial cells has been investigated (et al., 2008) [21]. Quantification of uncultured microorganisms by fluorescence microscopy and digital image analysis has been carried out (and Michael, 2007) [22]. The cell image analysis ontology using geometric and statistical features has investigated in et al. (2008) [23].

\section{Materials and Methods}

The separation of a dilute mixed population of microorganisms is done by using the spread plate technique. Thus, individual colonies are isolated. Aseptically transfer the loopful of mixed culture on the Nutrient Agar medium. Spread uniformly with the help of L-shaped spreader on the surface of medium plates. After spreading, incubate at $28^{\circ} \mathrm{C}$ for 30 days. After incubation, single colonies will appear on the Nutrient Agar media plates. Then pick up the colony and go further identification by using staining techniques. A smear of mixed culture of bacteria is deposited on a glass slide and thoroughly air-dried. The glass slide is examined under oil immersion with direct illumination in a 20 equipped with a 3 CCD Sony camera and connected to a PC. We have considered 512' 512 image sizes of 350 color images of 10,000magnifications for the present study and these are converted into images.

\section{Results and Discussions}

Let the horizontal or ' $\mathrm{x}$-axis' represent the number of days and the vertical or ' $\mathrm{y}$-axes represent the biomass variation on each of the days. We have a total of 15 readings recorded on alternate days through an entire month. The relationship between the number of days and amount of biomass produced is calculated. Person correlation is applied to the data set.

\section{Analysis}

Time series plot: The raw data tabulated contains two variables: ' $x$ ' (timestamp) and ' $y$ ' (a measurement of the parameter of interest). In all, there are 15 observations recorded through wet-lab studies. A plot of the parameter against time helps us study the trend in the data. 
DOI: https://dx.doi.org/10.26808/rs.ca.i10v6.01

Scatter plot: We plot the points on a graph to get a scatter diagram (i.e., the values of two parameters for corresponding time stamps). The scatter diagram helps us understand the relationship between the two parameters: whether the data is uncorrelated or the correlation is positive or negative.
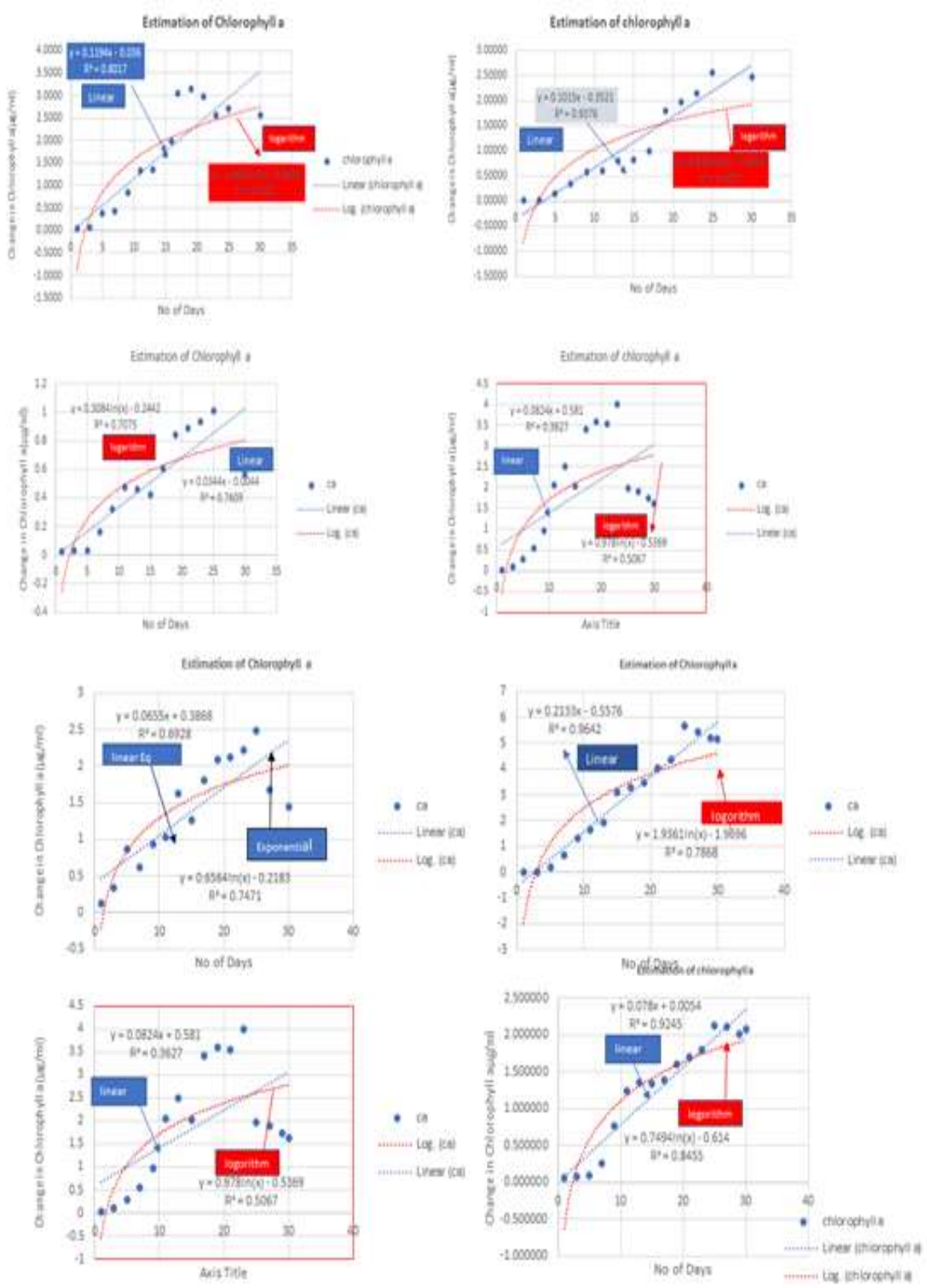

Figure 1: Scattershot showing the arrangement of data points on the map showing a positive correlation as the $\mathrm{R}^{2}$ value is near to one that signifies that the variables are closely associated with each other. A trend line is drawn on the data set plotted.

Time series plot: In our study, there is a positive correlation (observed as an upward trend in the plot) till a certain point of time (as the number of days increases the value of either biomass/chlorophyll) and then we see downward trend, a negative correlation is observed after a certain reading. This indicates that the value of variable ' $x$ ' that is a number of days increases the value of the biomass /chlorophyll a decrease as shown in the above scatter diagram figure1. 
DOI: https://dx.doi.org/10.26808/rs.ca.i10v6.01 International Journal of Computer Application (2250-1797)

Issue 10 Volume 6, November- December 2020
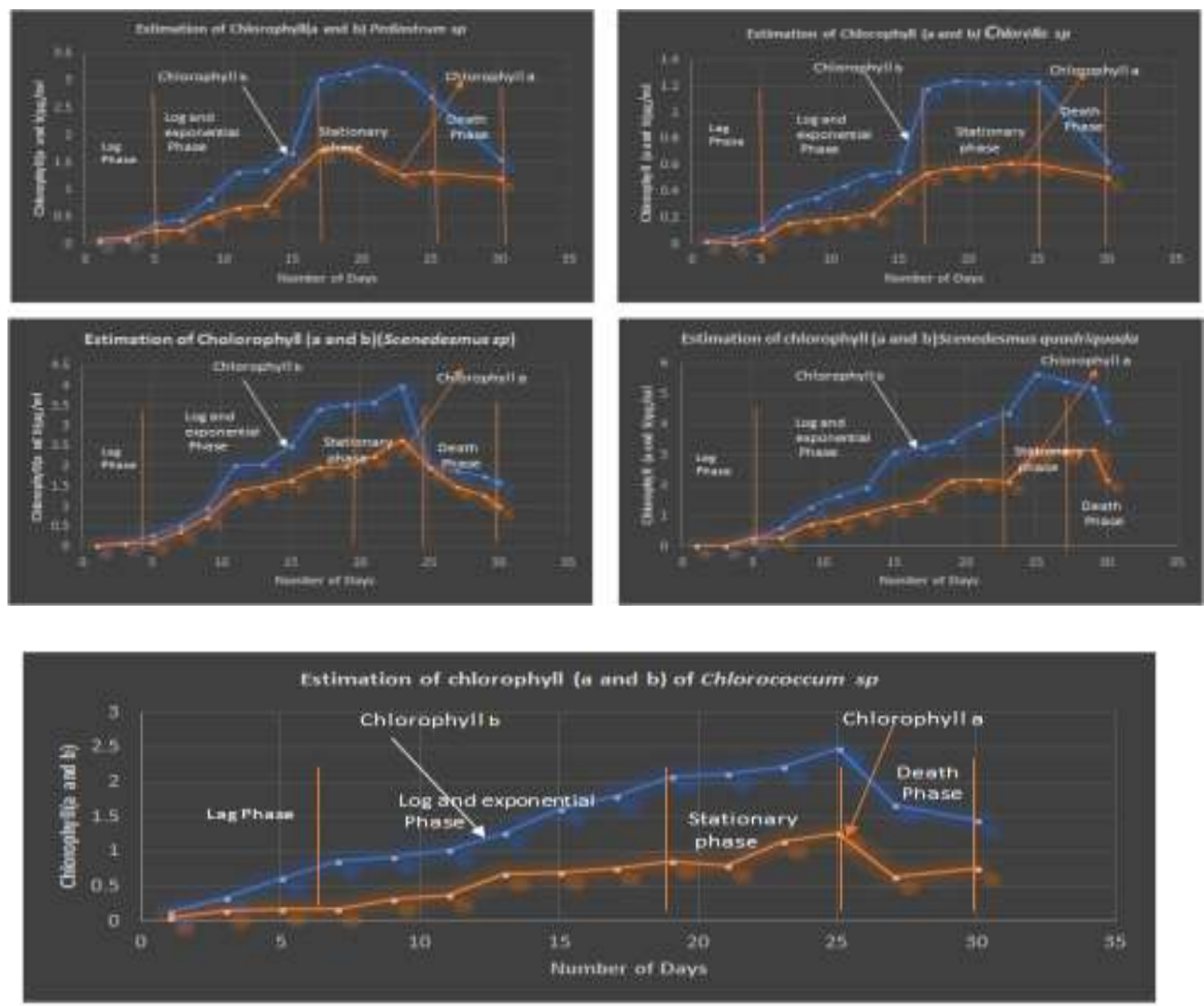

Figure 2:The above figure indicates the estimation of chlorophyll $\mathrm{a}$ and $\mathrm{b}$ moving from left to right (a)-(e) shows that in these species Pediastrum sp,Chlorellasp,Scenedesmus sp,Scenedesmus quadriquada sp, and Chlorococcum sp .

The above Figure 2 shows the production of chlorophyll a and $\mathrm{b}$ for the following species Pediastrum sp, Chlorella sp, Scenedesmus sp, Scenedesmus quadriquada, and Chlorococcum $s p$. We note that the yield of chlorophyll $\mathrm{b}$ is more than compared with the production of chlorophyll a. Further, both chlorophyll b,a increases exponentially during the exponential phase and slowly decline during the death phase and remain constant during the stationary phase of the growth curve. The production of chlorophyll is calculated for a month. The production of chlorophyll $\mathrm{b}$ is highest in the lifetime of Scenedesmus quadriquada sp.
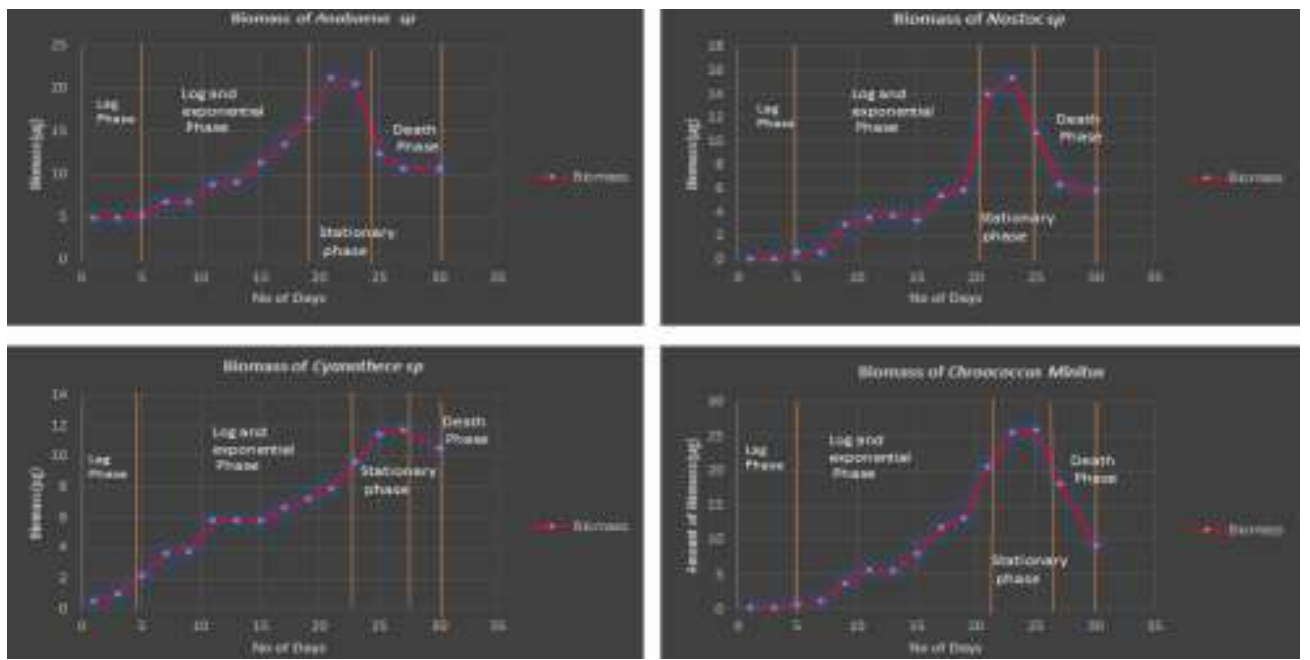

Figure 3: Graph represents the estimation of pigments chlorophyll a during growth curve species understudy 
Figure 3 shows plots of the content of chlorophyll a in Scenedesmus sp, Chlorella $s p$, Scenedesmus quadriquada sp, Pediastrum sp, and Chlorococcum sp and Figure 3 displays plots of the amount of chlorophyll-a in Nostos sp, Chroococcus sp and Anabaena sp. We note that among these, Chroococcus $s p$ produces the largest quantity of chlorophyll a.

In biological terms, initially, i.e., during the lag phase, the chlorophyll pigments do not show any promising increase in the values as the cells start to adjust the culture medium and environment. This is the lag phase. As time progresses, the quantity of pigment (chlorophyll a or b) is seen to increase, initially the increase is slow and at a point exponential - these are the lag and exponential phase of growth. After a certain duration of time, the quantity of pigments reaches a maximum and remains the same (seen as a plateau in the graph). Finally, the quantity of pigments decreases as the cells decline. This is the death phase. Thus, the entire growth cycle is divided into four phases: lag, log/ exponential, stationary and death as noted from Figure 3 using grids to separate each of the phases.

\section{Statistical Analysis based on Regression Analysis}

Regression analysis is used to identify the best line (or curve) through the set of data points. We have resorted to polynomial regression analysis to model our data. In this form of regression, the relationship between the independent variable $\mathrm{x}$ and dependent variable $\mathrm{y}$ is modelled as an $n^{\text {th }}$ degree polynomial. Polynomial regression fits using the least-squares method. The least-squares method minimizes the variance of the unbiased estimators of the coefficients, under the conditions of the Gauss-Markov theorem. The mathematical approach for the polynomial analysis can be expressed in the following general formula: $\mathrm{Y}=\mathrm{a}+\mathrm{bX}$

where $a=\frac{\left(\sum y\right)\left(\sum x^{\wedge} 2\right)-\left(\sum x\right)\left(\sum x y\right)}{n\left(\sum x^{2}-\left(\sum x\right)^{\wedge} 2\right.} b=\frac{n\left(\sum x y\right)-\left(\sum x\right)\left(\sum y\right)}{n\left(\sum x^{\wedge} 2\right)-\left(\sum x\right)^{\wedge} 2}$,

$y=p_{1} x^{n}+p_{2} x^{n-1}+\cdots+p_{n} x+p_{n+1}$

where $x$ is the independent variable, $y$ is the dependent variable, $n$ represents the degree of the polynomial and $p$ represents the coefficient of thepolynomial.R-squared - the square of the correlation coefficient - used to determine the strength of association between the two variables is given by -

$$
\mathrm{R}=\frac{n\left(\sum x y\right)-\left(\sum x\right)\left(\sum y\right)}{\sqrt{\left[n \sum x^{2}-\left(n \sum y 2-\left(\sum y\right)^{\wedge} 2\right]\right.}},
$$

where the numerator denoted residual sum of squares and the denominator indicates the total sum of squares. For the purpose of the present study, we study the relationships between chlorophyll a,b and biomass and also geometrical features such as area, major and minor axes, etc. Out of many parameters two are chosen at each time and in that one is taken as independent and the other as dependent variable for the plotting. A scatterplot provides a visualization of the relationship between two data sets. The dotted points on the graph represent the data values, which shows the visual representation of data. In the above plots, we are able to see that the points are closer and lie together forming a positive pattern, indicating that the correlation value is high. A positive correlation showing the number of days increases the amount of chlorophyll and even the biomass increases.

\section{Interpretation of Error Bars}

A line parallel to any one of the axes and passing through the points on the graph indicates the variation of the corresponding coordinate values at those points in the graphs. This is called an error bar. We have already established that the variation in values (across experiments) is on account of sampling of data from a population and is not significant. There is always a possibility that an experimental effect would have generated due to sampling errors. Here we consider $95 \%$ of confidence interval around the mean of 30 samples, if we 
DOI: https://dx.doi.org/10.26808/rs.ca.i10v6.01 International Journal of Computer Application (2250-1797) Issue 10 Volume 6, November- December 2020

repeat the experiment by phycologists' standards, p-values indicate the variations are on account of random experimental errors and not significant.
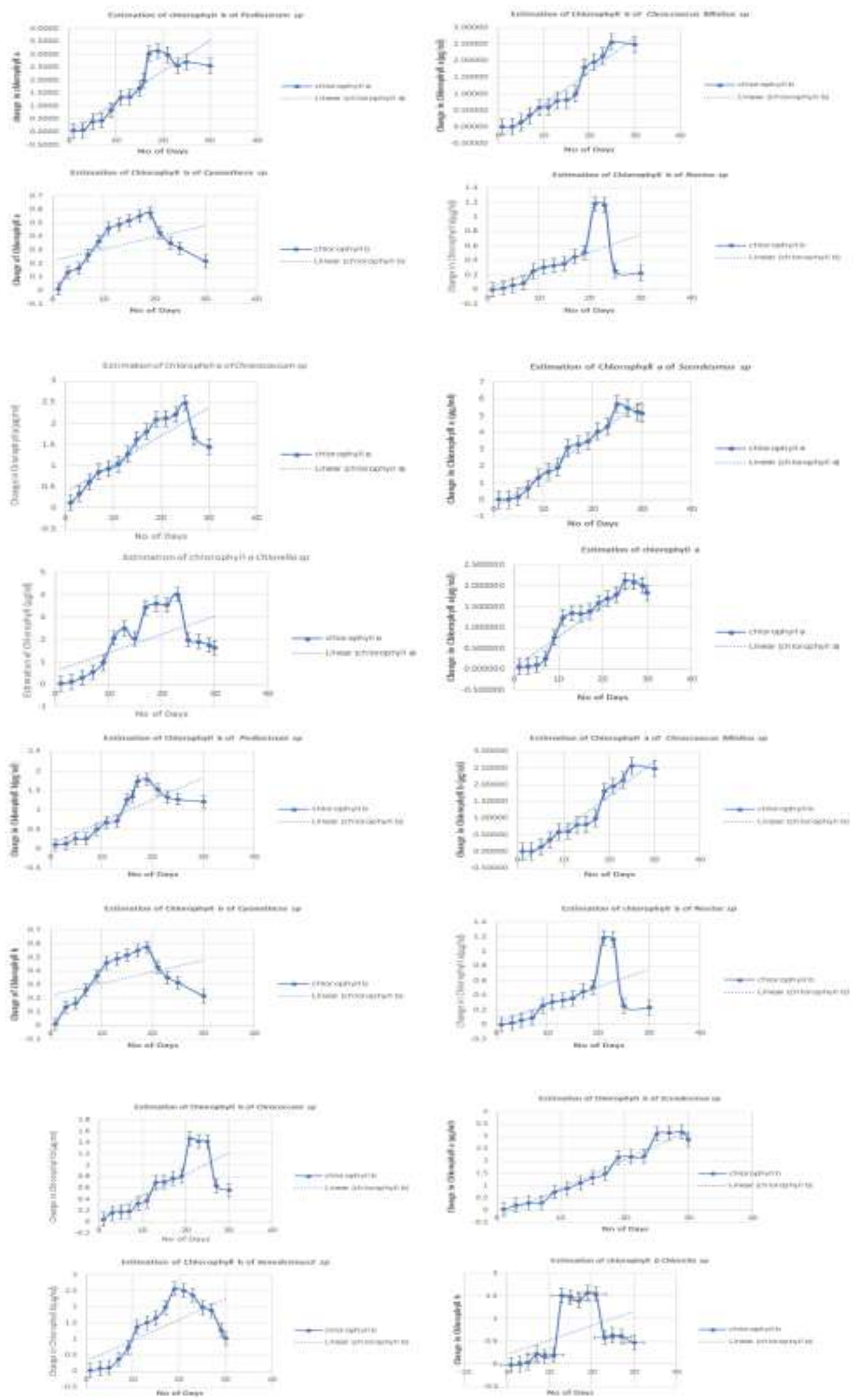

Figure 4:The following graph represents the error bars generated in the chlorophyll growth cycle of all the species and also a good fit line is drawn for these data points and horizontal error bars. These error bars are caused due to random experiment defects. 
Relating growth phases to time stamps (number of days) based on pigment content

In the graph Figure 4 it is clear that in almost all the above species growth curve shows that the chlorophyll a or chlorophyll $\mathrm{b}$ increases slowly between the $1^{\text {st }}$ to $5^{\text {th }}$ day and the cells are treated as normal (lag phase), from the $6^{\text {th }}$ day to $23^{\text {rd }} / 25^{\text {th }}$ day there is an exponential increase in either chlorophyll a or chlorophyll b content (log/ exponential phase), and $25^{\text {th }}$ day to $29^{\text {th }}$ day no change in the contents of the pigments the cell enters into grown-up stage (stationary phase) and the pigment decreases as the nutrients of the culture decreases the pigments also decreases from $29^{\text {th }}$ day onward (death phase). This quantification helps us train models and set up experiments for predicting the yield on a certain day or for computing the duration (number of days) it would take to achieve a certain yield of the pigment or biomass.

\section{Study of Biomass}
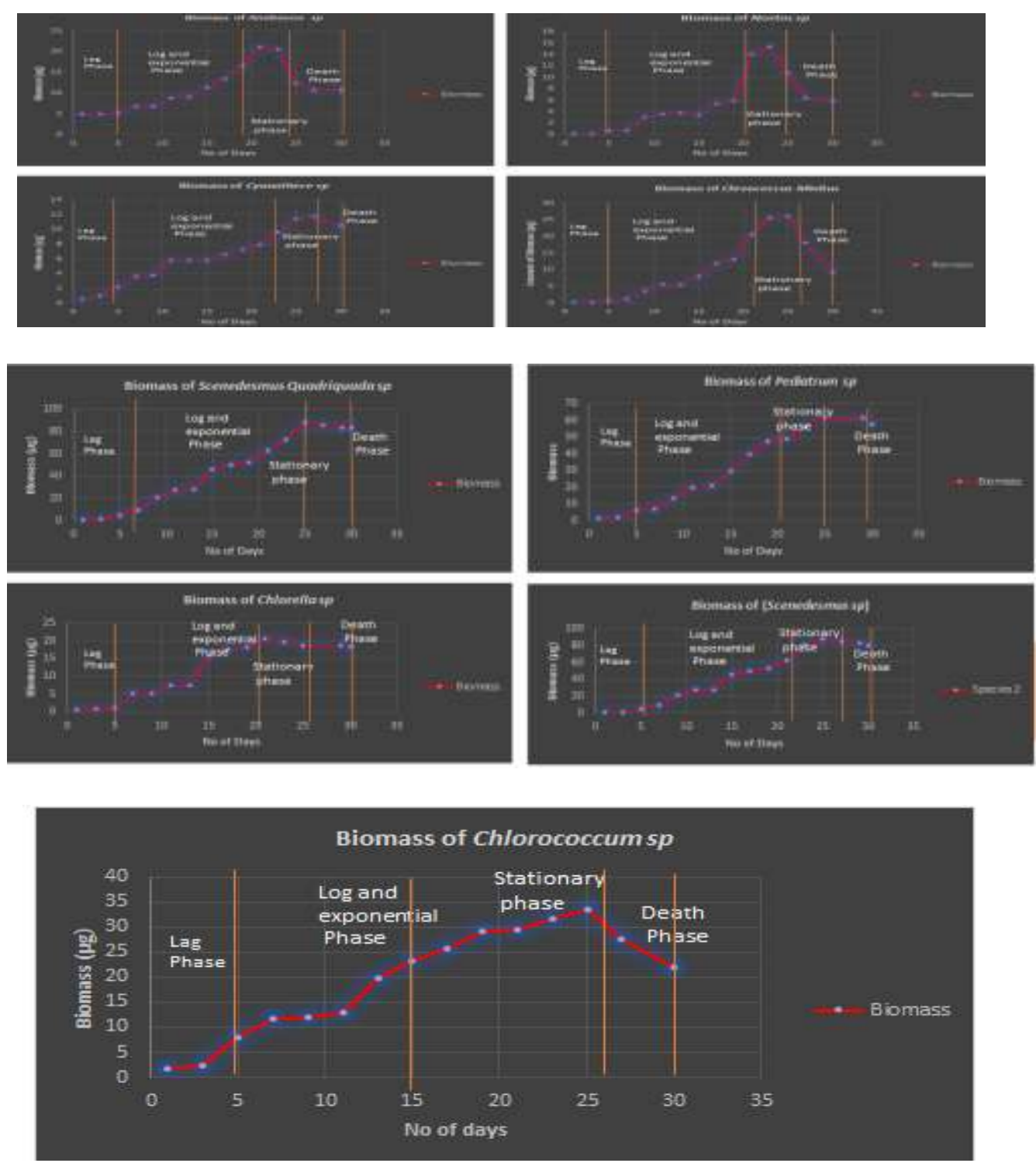

Figure 5 :Biomass estimation of various species in the growth curves

Another parameter that is estimated is biomass at an optimal temperature. As the number of days increases the contents of Biomass also increases as shown in Figure 5. Similar to the change in pigment values, changes in biomass are also viewed as four phases: first, during the 
DOI: https://dx.doi.org/10.26808/rs.ca.i10v6.01 International Journal of Computer Application (2250-1797) Issue 10 Volume 6, November- December 2020

Lag phase, there is a negligible increase in biomass value. As the day's progress cells enter into the Log and Exponential phase seen as a considerable increase in the biomass. Then, during the stationary phase, biomass remains unchanged. Finally, the value of biomass decreases as the cells decline; this is an indication of the death phase. Thus, the above graphs give a clear indication that the maximum biomass of the species is obtained during the exponential phase of the growth curve. This parameter is valuable in commercial applications in which algae are grown to extract yield such as Spirulina.

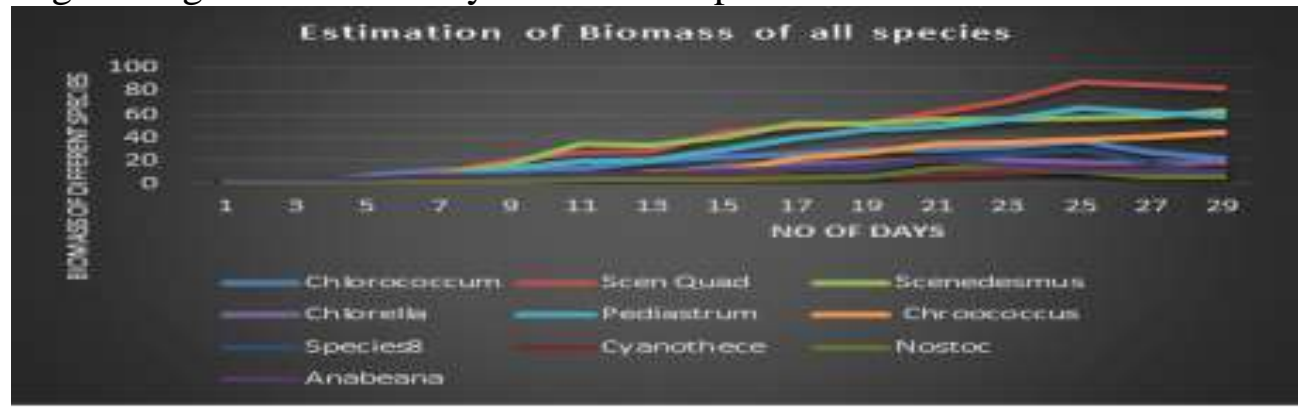

Figure 6: Consolidates estimation of biomass of the species Chlorococcum sp, Scenedesmus quadriquada,Scenedesmus sp, Pediastrum sp. and Cyanothecesp show maximum biomass of $37.59071 \times 10^{-3} \mathrm{mgl}^{-1}, 87.85819 \times 10^{-3} \mathrm{mgl}^{-1}, 65.27492 \times 10^{-3} \mathrm{mgl}^{-1}$ and $12.43144 \times 10^{-3} \mathrm{mgl}^{-}$ ${ }^{1}$ respectively on $25^{\text {th }}$ day of a growth cycle, whereas Chlorella $s p$, Nostos $s p$, and Anabaena $s p$ shows $21.6325 \times 10^{-3} \mathrm{mgl}^{-1}, 15.4356 \times 10^{-3} \mathrm{mgl}^{-1}$ respectively on $23^{\text {rd }}$ day of the growth cycle. Chroococcus $s p$ and Anabaena $s p$ shows $41.43 \times 10^{-3} \mathrm{mgl}^{-1}$ and $21.22096 \times 10^{-3} \mathrm{mgl}^{-1}$ on $27^{\text {th }}$ days and $21^{\text {st }}$ day of growth cycle respectively.

\section{The error bars generated in the chlorophyll growth cycle of all the species}
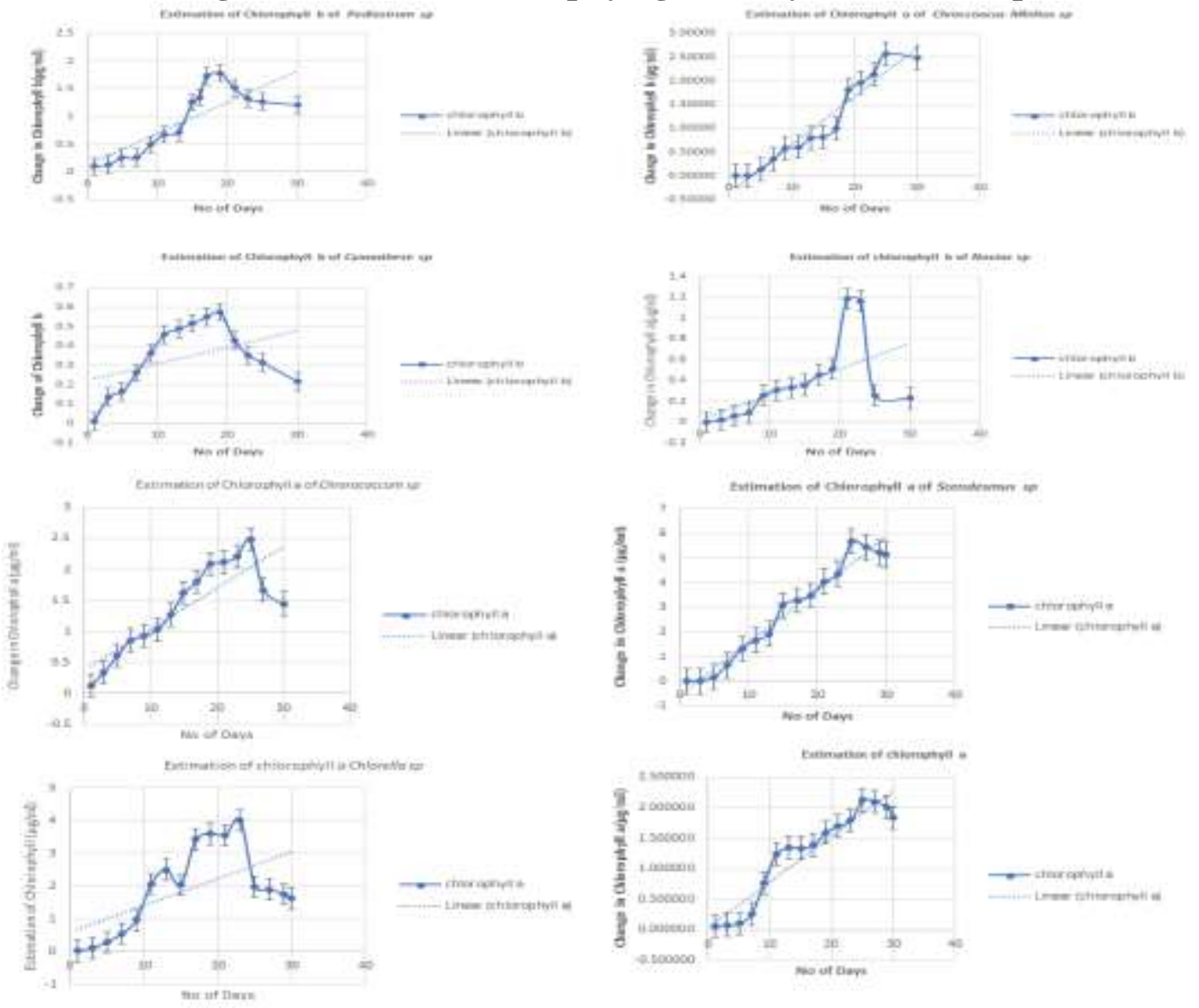
DOI: https://dx.doi.org/10.26808/rs.ca.i10v6.01

International Journal of Computer Application (2250-1797)

Issue 10 Volume 6, November- December 2020
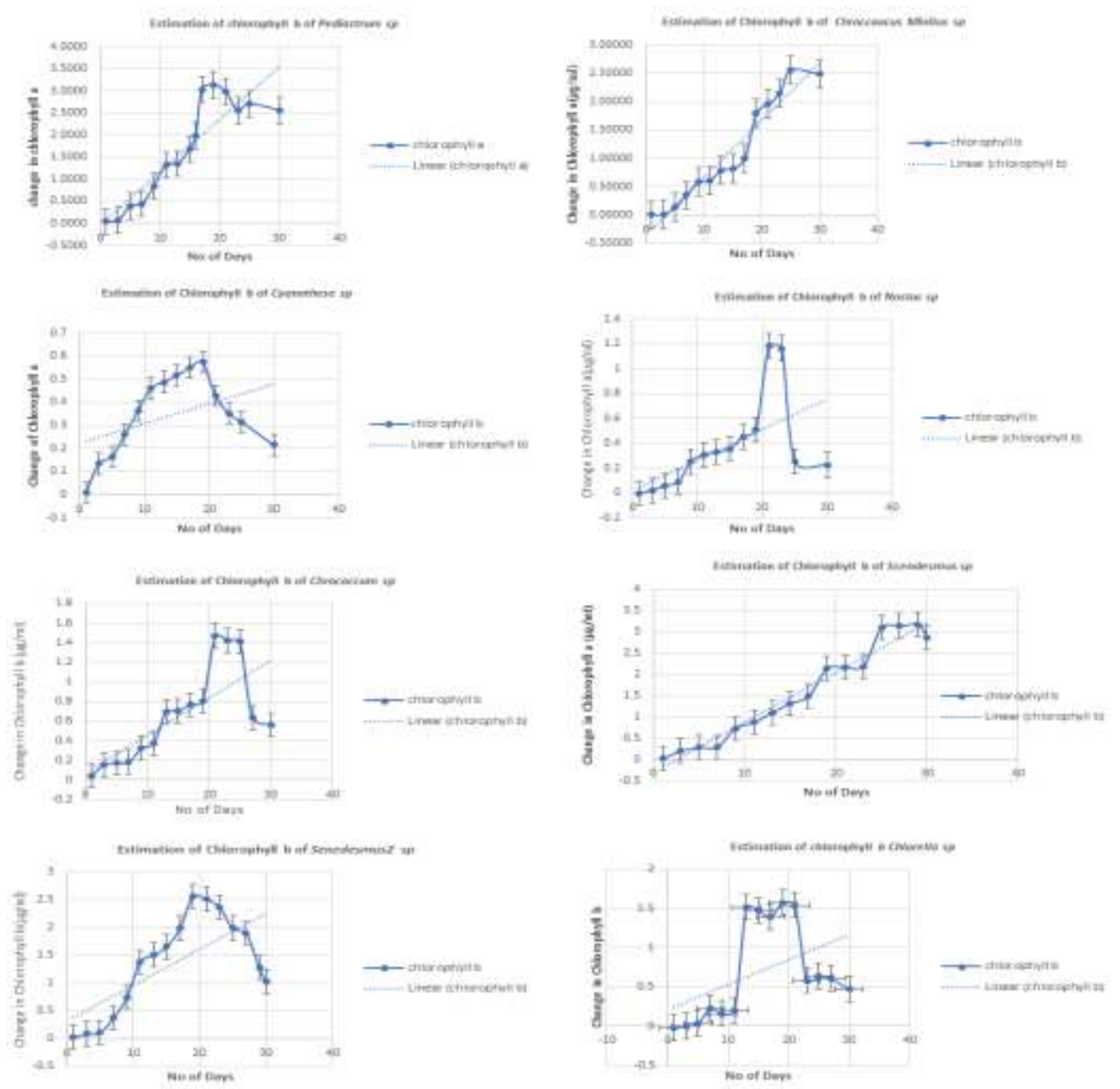

Figure 7:The following graph represents the error bars generated in the chlorophyll growth

cycle of all thespecies and also a good fit line is drawn for these data points and horizontalerror bars. These error bars are caused due to random experiment defects

\section{Forecasting of parameter values based on the training model}

Treating the data in the tables above as a regular time series (parameter measured against the number of days), we construct a model based on training data for which the value of the parameter for a corresponding timestamp is known. Then, we use test data for which a parameter has been measured for a given timestamp (say, Day 3) and attempt to estimate the value of the parameter for timestamp in the future (say, Day 17). In all such predictions, we notice the value of the parameter (such as biomass) predicted has a non-zero error when compared to the ground truth (viz., the value estimated by a biologist for that sample through wet lab experiments). Having already established there is a variation even in the 'ground truth' that is not significant, we determine using tests of significance that the deviation in the value predicted by the model from the ground truth is indeed a random 'error' that is acceptable within the margins of variation of biological samples. The graphs below plot the values obtained for forecasting parameter values based on the training models developed along with the ground truth values as measured by the biologist through wet-lab experiments. 
DOI: https://dx.doi.org/10.26808/rs.ca.i10v6.01

International Journal of Computer Application (2250-1797)

Issue 10 Volume 6, November- December 2020

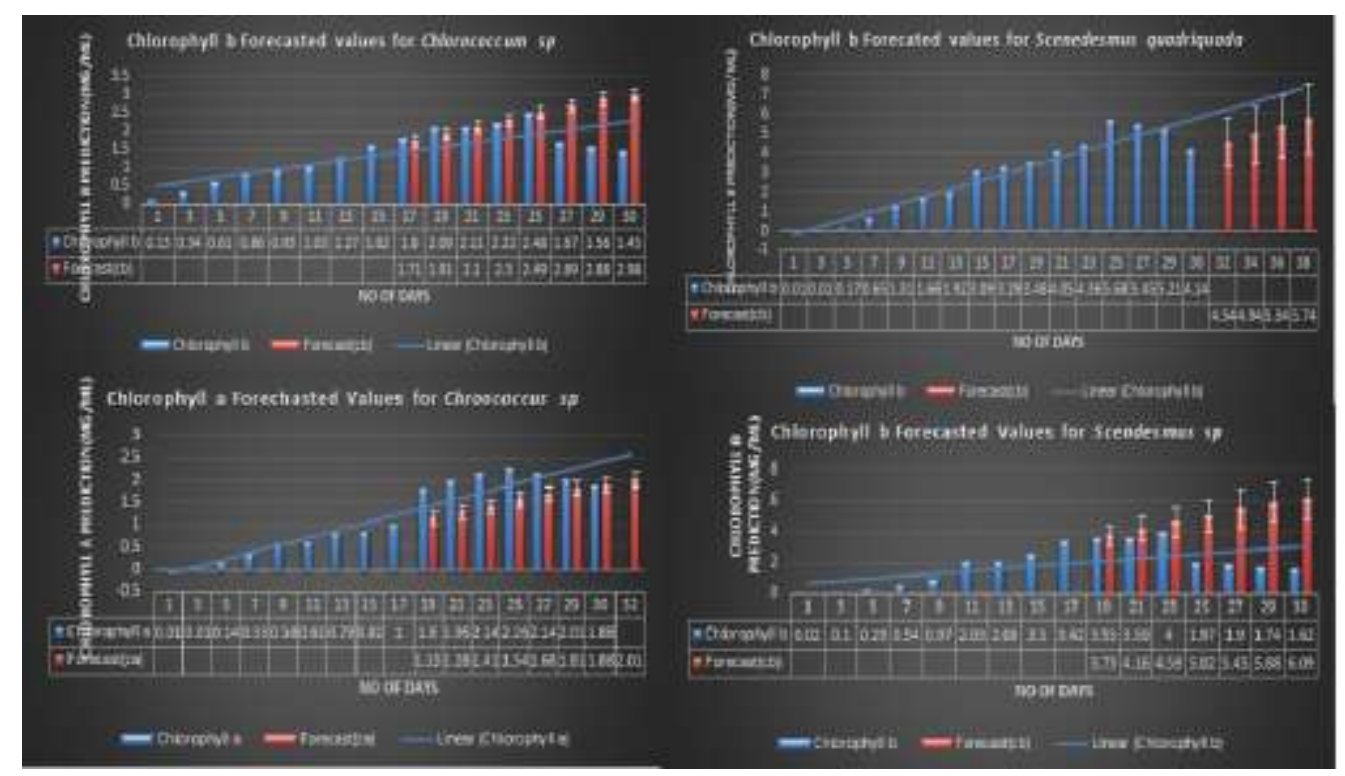

(a),(b),(c),(d) shows the forecast values of chlorophyll a, b in various species namely, Chlorococcum sp, Scendesmus sp, Scendesmus quadriquada, Pediastrum sp, Chroococcus sp

We observe that the forecast values are in good agreement with the ground truth for most of the species in the log/ exponential phase that is most important for commercial applications. Forecast values tend to remain high in the stationary phase and death phase because the linear model is not able to capture these dips suitably. We must use a non-linear model to capture these trends better. In the graphs below, we demonstrate the use of the autoregression integrated with moving average (ARIMA) model which incorporates corrections in the predicted values.

Forecasting of values is greatly improved with incorporating the moving average with the autoregression in the model. This model both short term changes captures the trend better than linear regression alone. We do notice that spurts in growth are not accurately modeled for some species such as with there being a noticeable difference in the predicted value and ground truth for a span of 3-5 days corresponding to the exponential growth phase, however, overall, the model outperforms simple linear regression for most of the species considered in this study.
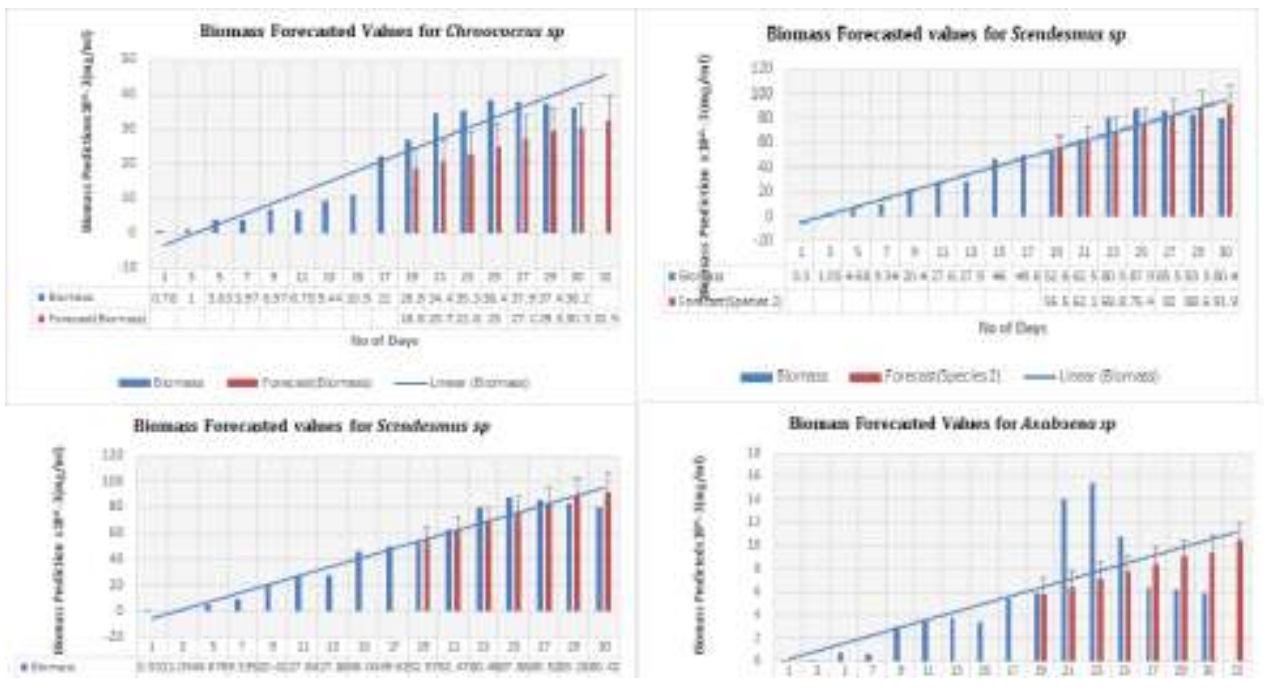

Figure 8:Forecasting biomass of various species 
These results are a proof-of-concept that parameters can be studied closely to be able to predict the yield for various species of microalgae for commercial applications where the yield of pigment or protein, etc., are of immense value. It is also clear that studying the changes in parameters helps us infer the growth stage of the microalgae and can be corroborated through imaging (using features extracted subsequently to automated segmentation). There is much scope for further research in understanding the values and building better time series models for forecasting, etc. Since this is the first study of its kind that has both wet-lab measurements in conjunction with imaging studies, to promote reproducible results, we have made the data set available in the public domain. Further, a biologist or typical end-user of a system such as this would not be interested in the technical nuances of mathematical models or tuning of parameters

\section{Conclusion}

This study presents an automated framework for interpreting large sets of visual information into a probabilistic phase profile of a cyanobacterial type. The procedure presented utilizes statistically based decisions methodology, with a minimal hard threshold in each of the steps throughout. The algorithm is robust to a variety of irregularities and variances between digital images and within images.

The results and discussion presented provide a probabilistic profiling per image group. Probabilistic phase profiling for digital images can provide a strong basis for further analysis and cyanobacterial type classification. Present study suggests a generic tool that supports the microbiologist in transforming and enhancing data into useful information for analysis. Objective and consistent processing are provided. In general, the tool we present provides an automated identifying and classification for the processing and analysis of large amounts of digital data. In much biological application, the amount of data is constantly increasing and a lot of inferences has to be derived so there is need to shift from manual work to an automated system is of increasing importance for efficient and accurate research and production.

\section{References}

[1] Gaston KJ, O’Neill MA. Automated species identification: why not? Philos Trans R Soc Lond B Biol Sci. 2004; 359(1444): 655-667, DOI: 10.1098/rstb.2003.1442, indexed in Pubmed: 15253351.

[2] Soberón J, Peterson AT. Biodiversity informatics: managing and applying primary biodiversity data. Philos Trans R Soc Lond B Biol Sci. 2004; 359(1444): 689-698, DOI: 10.1098/ rest.2003.1439, indexed in Pubmed: 15253354.

[3] Franco T, Wittmann D, Drauschke M, et al. Identification of Africanized honey bees through wing morphometrics: two fast and efficient procedures. Apidologie. 2008; 39(5): 488-494, DOI: 10.1051/apido:2008028.

[4] Kalafi EY, Tan WB, Town C, et al. Automated identification of Monogeneans using digital image processing and K-nearest neighbor approaches. BMC Bioinformatics. 2016; 17(Suppl 19): 511, DOI: 10.1186/s12859-0161376-z, indexed in Pubmed: 28155722.

[5] Leow LK, Chew LL, Chong VC, et al. Automated identification of copepods using digital image processing and artificial neural network. BMC Bioinformatics. 2015; 16 Suppl 18: S4, DOI: 10.1186/1471-2105-16-S18-S4, indexed in Pubmed: 26678287.

[6] Loos A, Ernst A. An automated chimpanzee identification system using face detection and recognition. EURASIP J Image Video Process. 2013; 2013(1): 49, DOI: 10.1186/16875281-2013-49. 
DOI: https://dx.doi.org/10.26808/rs.ca.i10v6.01 International Journal of Computer Application (2250-1797) Issue 10 Volume 6, November- December 2020

[7] Mehdipour Ghazi M, Yanikoglu B, Aptoula E. Plant identification using deep neural networks via optimization of transfer learning parameters. Neurocomputing. 2017; 235: 228235, DOI: 10.1016/j.neucom.2017.01.018.

[8] Salimi N, Loh KH, Dhillon SK, et al. Fully-automated identification of fish species based on otolith contour: using short-time Fourier transform and discriminant analysis (STFT-DA). PeerJ. 2016; 4: e1664, DOI: 10.7717/ peers.1664, indexed in Pubmed: 26925315.

[9] Underwood J, Dahlberg A, FitzPatrick S, et al. A STILE Project case study: The evaluation of a computer-based visual key for fossil identification. Assoc Learn Technol J. 1996; 4(2): 40-47, DOI: 10.1080/0968776960040205.

[10] Wen C, Guyer D, Li W. Local feature-based identification and classification for orchard insects. Biosystems $\quad$ Eng. 2009; 299-307, DOI: 10.1016/j.biosystemseng.2009.07.002.

[11] Yu X, Wang J, Kays R, et al. Automated identification of animal species in camera trap images. EURASIP J Image Video Process. 2013; 2013(1): 52, DOI: 10.1186/16875281-201352.

[12] Zhan M, Crane MM, Entchev EV, et al. Automated Processing of Imaging Data through Multi-tiered Classification of Biological Structures Illustrated Using Caenorhabditis elegans. PLoS Comput Biol. 2015; 11(4): e1004194, DOI: 10.1371//journal.pcbi.1004194, indexed in Pubmed: 25910032.

[13] Bunte K, Biehl M, Jonkman M, et al. Learning effective color features for content-based image retrieval in dermatology. Pattern Recognition. 2011; 44(9): 1892-1902, DOI: $10.1016 /$ j.patcog.2010.10.024.

[14] Singhai N, Shandilya S. A Survey On: "Content-Based Image Retrieval Systems". Int J Comput Appl. 2010; 4(2): 22-26, DOI: 10.5120/802-1139.

[15] Yue J, Li Z, Liu Lu, et al. Content-based image retrieval using color and texture fused features. Math Comput Model. 2011; 54(3-4): 1121-1127, DOI: 10.1016/j. mcm.2010.11.044.

[16] Trattner, S. and Greenspan, H. (2004) 'Automatic identification of bacterial types using statistical imaging methods', IEEE Transactions on Medical Imaging, Vol. 23, No. 7, pp.807-820.

[17] Nicholas, B., Åke, H., Johan, W., Rocio, C-H. and Peter, K.B. (1998) 'Rapid determination of bacterial abundance, biovolume, morphology, and growth by neural network-based image analysis', Applied and Environmental Microbiology, Vol. 64, No. 9, pp.3246-3255.

[18] Petra, P. (2001) 'Classification of HEp-2 cells using fluorescent image analysis and data mining',Medical Data Analysis, Springer-Verlag, INCS 2199, pp.219-224.

[19]Carolina, W., Joakim, L., Mikael, V., Ewert, B. and Lennart, B. (2002) 'Algorithms for cytoplasm segmentation of fluorescence-labeled cells', Analytical Cellular Pathology, Vol. 24, pp.101-111.

[20]Liu, J., Dazzo, F.B., Glagoleva, O., Yu, B. and Jain, A.K. (2001) 'CMEIAS: A Computer-Aided System for the Image Analysis of bacterial morphotypes in microbial communities', Microb. Ecol., Springer-Verlag, Vol. 41,pp.173-194

[21]Thomas, P., Josef, F., Martin, P. and Michaela, M.S. (2009) 'New image analysis tool to study biomass and morphotypes of three major bacterioplankton groups in an alpine lake', Aquatic Microbiol. Ecology, Vol. 54, pp.113-126.

[22] Holger, D. and Michael, W. (2007) 'Quantification of uncultured microogranisms by fluorescence microscopy and digital image analysis', Appl. Microbiol. Biotechnol. Springer, Vol. 75, pp.237-248.

[23] Hiremath, P.S. and Parashuram, B. (2010) 'Automatic identification and classification of bacilli bacterial cell growth phases', IJCA, Special Issue on RTIPPR, Vol. 1, pp.48-52. 